\title{
DESCRIPTIONS OF NEST ALGEBRAS
}

\author{
M. ANOUSSIS AND E. G. KATSOULIS
}

(Communicated by Paul S. Muhly)

\begin{abstract}
Every operator in the algebra of a continuous nest $\mathscr{N}$ can be factored as a product of two operators which belong to certain diagonal disjoint ideals of $\operatorname{Alg} \mathscr{N}$. This factorization leads to a new description of nest algebras.
\end{abstract}

The main result of this paper is that every operator in the algebra of a continuous nest can be factored as a product of two operators which belong to certain diagonal disjoint ideals of that algebra. As a consequence, a new description of nest algebras is given, in terms of factorization criteria.

Let $\mathscr{N}$ be a nest (i.e., a totally ordered complete set of projections containing 0 and $I$ ) on a separable Hilbert space $\mathscr{H}$. We denote by $\mathrm{Alg} \mathscr{N}$ the algebra of all operators in $B(\mathscr{H})$ that leave invariant every element of $\mathscr{N}$. Also, by $\mathscr{N}^{\prime}$ we will denote the diagonal of $\operatorname{Alg} \mathscr{N}$, i.e., the set of all operators in $\operatorname{Alg} \mathscr{N}$ such that their adjoints also belong to $\operatorname{Alg} \mathscr{N}$. Finally, we call $\mathscr{N}^{\prime \prime}$ to be the core of $\operatorname{Alg} \mathscr{N}$.

Ideals of nest algebras and in particular ideals which are diagonal disjoint have been studied by a number of authors [4, 5, 9]. For example, the Jacobson Radical $\mathscr{R}_{\mathcal{N}}$ of $\mathrm{Alg} \mathscr{N}$ is a diagonal disjoint two-sided ideal of $\mathrm{Alg} \mathscr{N}$ and one of the main results of [9] is a characterization of this ideal. Let us give some definitions.

We shall call a (not necessarily finite) set $\left\{P_{\alpha} \mid \alpha \in \mathscr{A}, \mathscr{A} \subseteq \mathbf{N}\right\}$ of intervals $P_{\alpha}=M_{\alpha}-N_{\alpha}, M_{\alpha}, N_{\alpha} \in \mathscr{N}$, of the nest $\mathcal{N}$, a partition of $\mathscr{N}$ if the intervals are pairwise orthogonal and the sum $\sum_{\alpha \in \mathscr{A}} P_{\alpha}=I$ in the strong operator topology. Given a nest $\mathscr{N}$, Larson's ideal $\mathscr{R}_{\mathscr{N}}^{\infty}$ is the collection of all operators $X$ in $\operatorname{Alg} \mathscr{N}$ for which, given $\varepsilon>0$, there exists a partition $\left\{P_{\alpha} \mid \alpha \in \mathscr{A}\right\}$ of $\mathscr{N}$ such that $\left\|P_{\alpha} X P_{\alpha}\right\|<\varepsilon$ for all $\alpha \in \mathscr{A}$.

If we restrict all partitions to be finite sets of intervals we will obtain the Jacobson Radical $\mathscr{R}_{\uparrow}$. of $\mathrm{Alg} \mathscr{N}$ [9]. Since, however, infinite partitions can arise in general, $\mathscr{R}_{\mathcal{N}}^{\infty}$ will usually contain $\mathscr{R}_{\mathcal{N}}$. The ideal $\mathscr{R}_{\mathcal{N}}^{\infty}$ plays an important role in the theory of nest algebras and their similarities [6]. Recently, J. Orr [11] proved that, for an arbitrary nest $\mathscr{N}, \mathscr{R}_{\mathcal{N}}^{\infty}$ is the greatest diagonal disjoint ideal of $\operatorname{Alg} \mathscr{N}$. One of the key lemmas in the proof of the above result

Received by the editors June 19, 1989.

1980 Mathematics Subject Classification (1985 Revision). Primary 47D25.

(C) 1990 American Mathematical Society $0002-9939 / 90 \$ 1.00+\$ .25$ per page 
follows. First, we need a definition:

Let $X \in \operatorname{Alg} \mathscr{N}$ and let $N$ be an element of $\mathscr{N}$. We say that $N$ belongs to the support of $X$ if and only if

$$
\inf \{\|(E-F) X(E-F)\| \mid E, \quad F \in \mathcal{N} ; E>N>F\}>0 .
$$

Proposition 1 ([11], Lemma 2.2). Let $\mathcal{N}$ be a continuous nest. Then there exists an infinite sequence $\left\{R_{n}\right\}_{n \in \mathbf{N}}$, of pairwise orthogonal care projections each of which has support equal to $\mathcal{N}$. There is also a bounded sequence of idempotents $Q_{n}=R_{n} Q_{n} R_{n}$ such that $Q_{n} \in \mathscr{R}_{\mathscr{N}}^{\infty}$ and such that $Q=\sum_{n=1}^{\infty} Q_{n}$ is a welldefined idempotent which has support equal to $\mathscr{N}$.

The following theorem, which is described in [7], is due to Larson-Pitts. We note that two idempotents $Q_{1}$ and $Q_{2}$ of $\operatorname{Alg} \mathcal{N}$ are said to be algebraically equivalent if there are operators $X, Y$ in $\operatorname{Alg} \mathcal{N}$ such that $X Y=Q_{1}$ and $Y X=Q_{2}$.

Theorem 2. Let $\mathcal{N}$ be a nest and let $Q_{1}, Q_{2}$ be idempotents in $\mathrm{Alg} \mathcal{N}$. Then $Q_{1}, Q_{2}$ are algebraically equivalent if and only if

$$
\operatorname{rank}(E-F) Q_{1}(E-F)=\operatorname{rank}(E-F) Q_{2}(E-F)
$$

for every $E>F$ in $\mathscr{N}$.

Let us now focus our attention on descriptions of nest algebras. There are several such descriptions in the literature. R. Loebl and P. Muhly [8] show that nest algebras are precisely the algebras of analytic operators with respect to certain groups of $*$-automorphisms of $B(\mathscr{H})$. J. Schue [10] describes nest algebras for which the diagonal is a maximal abelian algebra as the algebraic sum of the diagonal and a two-sided ideal defined in terms of certain boundedness conditions involving a certain derivation of $B(\mathscr{H})$. In the sequel we shall use a well-known description due to Deddens [2].

If $A=\int_{-\infty}^{+\infty} t d E_{A}(t)$ is a self-adjoint operator we call $\left\{E_{A}(t) \mid t \in \mathbf{R}\right\}$ the spectral nest of $A$ (see [1] for details). There is no loss of generality in assuming that every nest $\mathscr{N}$ is the spectral nest of a positive invertible operator. Indeed, if $f$ is a separating vector for $\mathscr{N}^{\prime \prime}, \mathscr{N}$ an arbitrary nest, then the operator

$$
A=\int \exp (\langle N f, f\rangle) d N
$$

which is well defined as a Riemman sum, is positive and invertible, and its spectral nest equals $\mathscr{N}$.

Theorem 3 (Deddens [2]). Let $A$ be a positive invertible operator with spectral nest $\mathscr{N}$. Then

$$
\operatorname{Alg} \mathscr{N}=\left\{X \in B(\mathscr{H}) \mid\left\|A^{m} X A^{-m}\right\| \leq\|X\|, m \in \mathbf{N}\right\} .
$$

The description of nest algebras that we give is slightly more complicated but also more detailed than Deddens'. We show that every operator in $\operatorname{Alg} \mathcal{N}, \mathcal{N}$ the spectral nest of a positive invertible operator $A$, factors as a product of two 
operators such that their half orbits, under the action of $A$, have special convergence properties (Theorem 8). Observe that it is not the case that the half orbits of every element of $\operatorname{Alg} \mathscr{N}$ have these convergence properties (Proposition 7). Hence our factorization result is the best that one can hope for.

In what follows, we will denote by $\mathscr{S}_{A}, A$ a positive invertible operator, the set of all operators $X$ in $B(\mathscr{H})$ such that the sequence $\left\{A^{m} X A^{-m}\right\}_{m \in \mathbf{N}}$ is strongly convergent to zero. Clearly

$$
\mathscr{S}_{A^{-1}}^{*}=\left\{X \in B(\mathscr{H}) \mid\left\{\left(A^{m} X A^{-m}\right)^{*}\right\}_{m \in \mathbf{N}} \text { is strongly convergent to zero }\right\} .
$$

is strongly convergent to zero $\}$. A more detailed analysis of $\mathscr{S}_{A}, \mathscr{S}_{A^{-1}}^{*}$ is needed.

Proposition 4. The set $\mathscr{S}_{A}, A$ a positive invertible operator with spectral nest $\mathscr{N}$, is a diagonal disjoint disjoint left ideal of $\mathrm{Alg} \mathscr{N}$, which is closed in the norm operator topology. Moreover, $\mathscr{N}^{\prime} \oplus \mathscr{S}_{A}$ is exactly the set of all operators $X$ in $B(\mathscr{H})$ such that the sequence $\left\{A^{m} X A^{-m}\right\}_{m \in N}$ is strongly convergent.

Proof. If $X$ belongs to $\mathscr{S}_{A}$, the uniform boundedness theorem shows that the sequence $\left\{A^{m} X A^{-m}\right\}_{m \in \mathbf{N}}$ is bounded in norm, hence by Theorem $3 X$ belongs to $\operatorname{Alg} \mathscr{N}$. Clearly, $\mathscr{S}_{A}$ is a diagonal disjoint left ideal of $\operatorname{Alg} \mathscr{N}$. Let us prove that it is norm closed.

Suppose $\left\{X_{k}\right\}_{k \in \mathbf{N}} \rightarrow X$ and $X_{k} \in \mathscr{S}_{A}, k \in \mathbf{N}$. Then $X_{k} \in \operatorname{Alg} \mathscr{N}$ and $X \in \operatorname{Alg} \mathscr{N}$, so, using Theorem 3 again, we have,

$$
\begin{aligned}
\left\|A^{m} X A^{-m} f\right\| & \leq\left\|A^{m}\left(X-X_{k}\right) A^{-m} f\right\|+\left\|A^{m} X_{k} A^{-m} f\right\| \\
& \leq\left\|X-X_{k}\right\|\|f\|+\left\|A^{m} X_{k} A^{-m} f\right\|, \quad f \in \mathscr{H} .
\end{aligned}
$$

The conclusion follows in the obvious way from this inequality.

Finally, let $X$ be an operator such that $\left\{A^{m} X A^{-m}\right\}_{m \in \mathbf{N}}$ is strongly convergent to an element of $B(\mathscr{H})$, namely $Y$. Then,

$$
A Y A^{-1}=A\left(\lim _{m} A^{m} X A^{-m}\right) A^{-1}=Y
$$

and so $A Y=Y A$ which implies $Y \in \mathscr{N}^{\prime}$. Moreover, $X-Y$ belongs to $\mathscr{S}_{A}$ and the proof of the proposition is complete.

Lemma 5. Let $A$ be a positive invertible operator with spectral nest $\mathscr{N}$. If $R$ is the direct sum of an infinite sequence $\left\{R_{n}\right\}_{n \in \mathbf{N}}$ of pairwise orthogonal core projections and $X=X R$ is an operator in Alg $\mathscr{N}$ such that $X R_{n} \in \mathscr{S}_{A}$, for every $n \in \mathbf{N}$, then $X$ belongs to $\mathscr{S}_{A}$.

Proof. Given $\varepsilon>0$ and a unit vector $f$ in $\mathscr{H}$, there exists $k \in \mathbf{N}$ such that $\left\|\sum_{n=k+1}^{\infty} R_{n} f\right\|<\varepsilon$. Hence,

$$
\begin{aligned}
\left\|A^{m} X A^{-m} f\right\| & \leq\left\|A^{m} X A^{-m}\left(\sum_{n=1}^{k} R_{n} f\right)\right\|+\left\|A^{m} X A^{-m}\left(\sum_{n=k+1}^{\infty} R_{n} f\right)\right\| \\
& \leq \sum_{n=1}^{k}\left\|A^{m}\left(X R_{n}\right) A^{-m} f\right\|+\|X\| \varepsilon,
\end{aligned}
$$

and the conclusion follows. 
Proposition 6. Let $A$ be a positive invertible operator with spectral nest $\mathscr{N}$. Then $\mathscr{S}_{A}$ contains the ideal $\mathscr{R}_{\mathscr{N}}^{\infty}$. Moreover, if $\mathscr{N}$ is continuous and $Q$ is the idempotent described in Proposition 1, then $Q$ belongs to $\mathscr{S}_{A}$. Hence, $\mathscr{S}_{A}$ equals $\mathscr{R}_{\mathscr{N}}^{\infty}$ if and only if $\mathscr{N}$ is completely atomic (i.e., the minimal intervals of $\mathscr{N}$ span the whole space $\mathscr{H}$ ).

Proof. We claim that every operator of the form $E X(I-E)$, where $E \in \mathscr{N}$ and $X \in B(\mathscr{H})$, belongs to $\mathscr{S}_{A}$.

First, we suppose that $E=E_{+} \equiv \wedge\{F \in \mathscr{N} \mid F>E\}$. Then, given $\varepsilon>0$ and a unit vector $f$ in $\mathscr{H}$, there exists $F>E$ in $\mathscr{N}$ such that $\|(F-E) f\| \leq$ $\varepsilon$. Since $F$ and $E$ are distinct there are positive numbers $\lambda<\mu$ such that $E_{A}(\lambda)=E$ and $E_{A}(\mu)=F$. Then,

$$
\begin{aligned}
\left\|A^{m} E X(I-E) A^{-m} f\right\|= & \left\|A^{m} E X(I-E) A^{-m}(F-E) f\right\| \\
& +\left\|A^{m} E X(I-E) A^{-m}(I-F) f\right\| \\
\leq & \left\|A^{m} X A^{-m}\right\| \varepsilon+\left\|A^{m} E\right\|\|X\|\left\|(I-F) A^{-m}\right\| \\
\leq & \|X\| \varepsilon+(\lambda / \mu)^{m}\|X\|
\end{aligned}
$$

and the conclusion follows.

If $E \neq E_{+}$, let $\lambda<\mu$ be positive numbers such that $E_{A}(\lambda)=E$ and $E_{A}(\mu)=E$. Then

$$
A^{m}=\int_{0}^{\lambda} t^{m} d E_{A}(t)+\int_{\mu}^{\infty} t^{m} d E_{A}(t), \quad m \in \mathbf{N} .
$$

So, $\left\|A^{m} E\right\|=\left\|\int_{0}^{\lambda} t^{m} d E_{A}(t)\right\| \leq \lambda^{m},\left\|(I-E) A^{-m}\right\| \leq \mu^{-m}$ and the conclusion follows by imitating the above proof.

Let $\left\{P_{\alpha} \mid P_{\alpha}=F_{\alpha}-E_{\alpha}, \alpha \in \mathscr{A}\right\}$ be a partition of $\mathscr{N}$ and $X$ be a operator in Alg $\mathscr{N}$ such that $X P_{\alpha}=E_{\alpha} X P_{\alpha}$ for every $\alpha \in \mathscr{A}$. Then the above claim shows that $X P_{\alpha} \in \mathscr{S}_{A}, \alpha \in \mathscr{A}$, and so, by Lemma $5, X$ belongs to $\mathscr{S}_{A}$. Since every operator in $\mathscr{R}_{\mathscr{N}}^{\infty}$ is the norm limit of a sequence of operators of the above form, Proposition 4 implies that $\mathscr{S}_{A}$ contains $\mathscr{R}_{\mathcal{N}}^{\infty}$.

We now claim that if $\mathscr{N}$ is completely atomic, then $\mathscr{R}_{\mathscr{N}}^{\infty}=\mathscr{S}_{A}$. Indeed, if $X \in \mathscr{S}_{A}$ and $P$ is an arbitrary atom of $\mathscr{N}$ then $P X P \in \mathscr{N}^{\prime}$ and so $P X P=$ $A^{m} P X P A^{-m}=P A^{m} X A^{-m} P$, for every $m \in \mathbf{N}$. Thus $P X P=0$, which shows that $X \in \mathscr{R}_{\mathcal{1}}^{\infty}$.

If $\mathscr{N}$ is a continuous nest Lemma 5 shows that the idempotent $Q$ described in Proposition 1 is contained in $\mathscr{S}_{A}$. Clearly, $Q$ does not belong to $\mathscr{R}_{\mathcal{N}}^{\infty}$ and so $\mathscr{R}_{\mathscr{N}}^{\infty}$ is strictly contained in $\mathscr{S}_{A}$. For the general case, if $\mathscr{N}$ is not completely atomic, a simple reduction to the continuous part of $\mathscr{N}$ shows the desired result.

Remarks. (a) If $\mathscr{N}^{\perp} \equiv\{I-N \mid N \in \mathscr{N}\}$ then $\mathscr{R}_{1^{\perp}}^{\infty}=\left(\mathscr{R}_{1}^{\infty}\right)^{*}$ and so Proposition 6 shows that $\mathscr{S}_{A^{-1}}^{*}$ contains the ideal $\mathscr{R}_{1}^{*}$. Moreover, since the idempotent $Q$ is a direct sum of operators that belong to $\mathscr{R}_{1}^{\infty}$, the above observation implies that $Q^{*} \in \mathscr{S}_{A^{-1}}$. 
(b) Combining Proposition 6 with the Erdos Density Theorem [3, Theorem $3]$ one can prove that if $A$ is a positive operator with continuous spectral nest, then the ideals $\mathscr{S}_{A}, \mathscr{S}_{A^{-1}}^{*}$ are never closed in the strong operator topology. Hence, Lemma 5 is not obvious.

We are now in position to state our main results. We begin our exposition with a characterization of the algebras of completely atomic nests.

Proposition 7. Let $A$ be a positive invertible operator with spectral nest $\mathcal{N}$. Then

$$
\text { Alg } \mathscr{N}=\left\{X \in B(\mathscr{H}) \mid\left\{A^{m} X A^{-m}\right\}_{m \in \mathbf{N}} \text { is strongly convergent }\right\}
$$

if and only if $\mathcal{N}$ is completely atomic.

Proof. Let $A$ be a positive operator with completely atomic spectral nest $\mathscr{N}$. Then, by Proposition 6, Alg $\mathscr{N}=\mathscr{N}^{\prime} \oplus \mathscr{R}_{\mathscr{N}}^{\infty}=\mathscr{N}^{\prime} \oplus \mathscr{S}_{A}$, which implies that $\mathrm{Alg} \mathscr{N}$ is exactly the set of all operators $X$ in $B(\mathscr{H})$ such that the sequence $\left\{A^{m} X A^{-m}\right\}_{m \in \mathbf{N}}$ converges in the strong operator topology (Proposition 3 ).

Conversely, let $A$ be a positive invertible operator with spectral nest $\mathscr{N}$, such that $\operatorname{Alg} \mathscr{N}=\mathscr{N}^{\prime} \oplus \mathscr{S}_{A}$. We will prove that $\mathscr{N}$ is completely atomic. Indeed if it is not completely atomic then there exists a projection $P \in \mathcal{N}^{\prime}$ such that the compressed nest $P \mathscr{N} \equiv\{P N \mid N \in \mathscr{N}\}$, acting on the Hilbert space $P(\mathscr{H})$, is a multiplicity free continuous nest. Moreover, $\mathrm{Alg} P \mathscr{N}=$ $P($ Alg $\mathscr{N}) P=(P \mathscr{N})^{\prime} \oplus \mathscr{S}_{P A P}$, which implies that $\mathscr{S}_{P A P}$ is a two-sided diagonal disjoint ideal of $\operatorname{Alg} P \mathscr{N}$. Since $(P \mathscr{N})^{\prime}$ is abelian the ideal $\mathscr{S}_{P A P}$ contains the commutator ideal of $\operatorname{Alg} P \mathscr{N}$ (that is to say the smallest ideal containing all the commutators $Y X-X Y$ of operators in $\mathrm{Alg} P \mathscr{N}$ ). However in [6, Proposition 3.5], Larson shows that the commutator ideal for the algebra of a continuous nest is the whole algebra, and thus $\mathscr{S}_{P A P}=\operatorname{Alg} P \mathscr{N}$, which is absurd.

How can Proposition 7 be generalized for an arbitrary positive invertible operator $A$ ? Clearly, if the spectral nest $\mathscr{N}$ of $A$ is not completely atomic then there exists an operator $X$ in $\operatorname{Alg} \mathscr{N}$ such that the sequence $\left\{A^{m} X A^{-m}\right\}_{m \in \mathbf{N}}$ does not converge in the strong operator topology. In spite of this, our main result guarantees that every such operator can be factored as the product of two operators $Y, Z$ in $\operatorname{Alg} \mathscr{N}$ such that $\left\{A^{m} Y A^{-m}\right\}_{m \in \mathbf{N}},\left\{\left(A^{m} Z A^{-m}\right)^{*}\right\}_{m \in \mathbf{N}}$ are strongly convergent.

Theorem 8. Let $A$ be a positive invertible operator with spectral nest $\mathscr{N}$. Then, an operator $X$ belongs to $\mathrm{Alg} \mathscr{N}$ if and only if it can be factored as a product $X=Y Z$, where $Y \in \mathscr{N}^{\prime} \oplus \mathscr{S}_{A}, Z \in \mathscr{N}^{\prime} \oplus \mathscr{S}_{A^{-1}}^{*}$.

Proof. Clearly every product of the form $X=Y Z$, where $Y \in \mathscr{N}^{\prime} \oplus \mathscr{S}_{A}$, $Z \in \mathscr{N}^{\prime} \oplus \mathscr{S}_{A^{-1}}^{*}$, belongs to $\operatorname{Alg} \mathscr{N}$.

Conversely let $X$ be an arbitrary element of $\operatorname{Alg} \mathscr{N}$. We will prove that $X$ admits the desired factorization.

Let $I-P$ be the span of all atoms of $\mathscr{N}$. Then, the nest $P \mathcal{N}$ is continuous and hence there exists an idempotent $Q_{0} \in \operatorname{Alg} P \mathscr{N}$ satisfying the properties 
described in Proposition 1. If $Q=Q_{0} \oplus 0$, with respect to the resolution $\mathscr{H}=P(\mathscr{H}) \oplus(I-P)(\mathscr{H})$, then Proposition 6 shows that the idempotent $Q$ lies in $\mathscr{S}_{A} \cap \mathscr{S}_{A^{-1}}^{*}$.

An immediate application of Theorem 2 shows that there exist operators $S, T$ in $\operatorname{Alg} \mathcal{N}(S=P S P, T=P T P)$ such that $Q=T S$ and $P=S T$ (we note that every nonzero idempotent lying in the algebra of a continuous nest is of infinite rank). If $Y \equiv X(I-P)+X S Q$ and $Z \equiv I-P+Q T$ then,

$$
Y Z=X(I-P)+X S Q T=X(I-P)+X(S T)^{2}=X .
$$

Moreover, since $P \in \mathscr{N}^{\prime}$ and $Q T \in \mathscr{S}_{A^{-1}}^{*}$, we have that $Z \in \mathscr{N}^{\prime} \oplus \mathscr{S}_{A^{-1}}^{*}$. On the other hand, Proposition 7 shows that $(I-P) X(I-P)$ lies in $\mathscr{N}^{\prime} \oplus \mathscr{S}_{A}$. Since, by Lemma 5, $P X(I-P) \in \mathscr{S}_{A}$ we have that the operator $X(I-P)$ belongs to $\mathscr{N}^{\prime} \oplus \mathscr{S}_{A}$. Clearly $X S Q \in \mathscr{S}_{A}$ and hence $Y \in \mathscr{N}^{\prime} \oplus \mathscr{S}_{A}$, which proves the theorem.

From the proof of the above theorem follows immediately that if the spectral nest $\mathscr{N}$ of $A$ is continuous then every operator $X$ in $\operatorname{Alg} \mathscr{N}$ can be factored as a product $X=Y Z$, where $Y, Z$ belong to $\mathscr{S}_{A}, \mathscr{S}_{A^{-1}}^{*}$, respectively.

Corollary 9. Let $A$ be a positive invertible operator with spectral nest $\mathcal{N}$. Then $\mathscr{N}$ is continuous if and only if every element $X$ of $\operatorname{Alg} \mathscr{N}$ admits a factorization $X=Y Z$, where $Y, Z$ belong to $\mathscr{S}_{A}, \mathscr{S}_{A^{-1}}^{*}$, respectively.

Remarks. It is remarkable that our description of nest algebras depends only on the choice of nest $\mathscr{N}$; it is independent of the choice of the positive invertible operator $A$ whose spectral nest equals $\mathscr{N}$. In fact, if $\mathscr{N}$ is a completely atomic nest then all the ideals $\mathscr{S}_{A}$, as $A$ varies over the positive invertible operators with spectral nest $\mathscr{N}$, are equal to Larson's ideal $\mathscr{R}_{\mathcal{N}}^{\infty}$. Unfortunately, it is not known to us how the ideals $\mathscr{S}_{A}$ vary with $A$ when the nest $\mathscr{N}$ is not completely atomic.

Moreover, there is another open question related to the one above. First, we give a definition: Let $\mathscr{N}$ be an arbitrary nest. We define $\mathscr{S}_{\mathscr{N}}$ to be the set of all operators $X$, in $\operatorname{Alg} \mathscr{N}$ with the property that given any strong operator topology neighborhood of zero, there is a finite partition $\left\{P_{\alpha} \mid \alpha \in \mathscr{A}\right\}$ of $\mathscr{N}$ such that $\sum_{\alpha \in \mathscr{A}} P_{\alpha} X P_{\alpha}$ lies in this neighborhood.

The set $\mathscr{S}_{\mathcal{N}}$ is a norm closed, diagonal disjoint, left ideal of Alg $\mathscr{N}$. Moreover, $\mathscr{S}_{\mathcal{N}}$ contains Larson's ideal $\mathscr{R}_{\mathcal{N}}^{\infty}$ (see [5] for a proof) and hence a reworking of the proof of Theorem 8 shows that the statement of that theorem remains true if $\mathscr{S}_{A}, \mathscr{S}_{A^{-1}}^{*}$ are replaced by $\mathscr{S}_{\mathscr{N}}, \mathscr{S}_{\mathcal{N}^{\perp}}^{*}$ respectively. We don't know how the ideal $\mathscr{S}_{\mathscr{N}}$ is related to the ideals $\mathscr{S}_{A}$, where $A$ ranges over the positive invertible operators with spectral nest $\mathscr{N}$. Does there exist a positive invertible operator $A$ such as $\mathscr{S}_{A}$ equals $\mathscr{S}_{\mathcal{N}}$ ? Clearly, an answer to the above questions would give a better understanding of our factorization results.

If someone wants to use weak operator topology criteria in order to describe nest algebras, then the following is the best that can be said. 
Proposition 10. Let $A$ be a positive invertible operator with spectral nest $\mathscr{N}$. Then $\operatorname{Alg} \mathscr{N}$ is exactly the set of all operators $X$ in $B(\mathscr{H})$ for which the sequence $\left\{A^{m} X A^{-m}\right\}_{m \in \mathbf{N}}$ has a weakly convergent subsequence.

Proof. If $X \in \operatorname{Alg} \mathscr{N}$, then the sequence $\left\{A^{m} X A^{-m}\right\}_{m \in \mathbf{N}}$ is bounded in norm, hence it has a weakly convergent subsequence. For the converse, let $X$ be an operator such that $\left\{A^{m} X A^{-m}\right\}$ has a weakly convergent subsequence; namely, $\left\{A^{m_{k}} X A^{-m_{k}}\right\}_{k \in \mathbf{N}}$. Hence, by the uniform boundedness theorem there exists a constant $C>0$ such that $\left\|A^{m_{k}} X A^{-m_{k}}\right\| \leq C, k \in \mathbf{N}$. We will prove that $X$ belongs to $\operatorname{Alg} \mathscr{N}$.

Let $E=E_{A}(\lambda), \lambda \in \mathbf{R}^{+}$, be an arbitrary element of $\mathscr{N}$. Then, for every $\mu>\lambda$,

$$
\begin{aligned}
\left\|\left(I-E_{A}(\mu)\right) X E_{A}(\lambda)\right\| & \leq\left\|A^{-m_{k}}\left(I-E_{A}(\mu)\right)\right\|\left\|A^{m_{k}} X A^{-m_{k}}\right\|\left\|E_{A}(\lambda) A^{m_{k}}\right\| \\
& \leq C(\lambda / \mu)^{m_{k}} .
\end{aligned}
$$

Hence $\left(I-E_{A}(\mu)\right) X E_{A}(\lambda)=0$ for every $\mu>\lambda$. The conclusion now follows from the fact that $E_{A}(\mu) \downarrow E_{A}(\lambda)$ as $\mu \downarrow \lambda$.

\section{ACKNOWLEDGMENT}

We would like to express appreciation to Dr. J. Orr for the fruitful discussions we had during our visit to Lancaster.

\section{REFERENCES}

1. J. B. Conway, A course in functional analysis, Graduate Texts in Mathematics, no. 96, Springer-Verlag, New York, 1985.

2. J. A. Deddens, Another description of nest algebras, in Hilbert Space Operators, Lecture Notes in Math., vol. 693, Springer-Verlag, New York, 1978.

3. J. A. Erdos, Operators of finite rank in nest algebras, J. London Math. Soc. 43 (1968), 47-63.

4. __ On some ideals of nest algebras, Proc. London Math. Soc. 44 (1982), 143-160.

5. A. Hopenwasser, Hypercasual linear operators, SIAM J. Control Optim. 22 (1984), 911-919.

6. D. R. Larson, Next algebras and similarity transformations, Ann. Math. 121 (1985), 409427.

7. __ Some recent progress in nest algebras, preprint, Dept. of Math., Texas A\&M University.

8. R. I. Loebl and P. S. Muhly, Analyticity and flows in von Neumann algebras, J. Funct. Anal. 29 (1978), 214-252.

9. J. R. Ringrose, On some algebras of operators, Proc. London Math. Soc. 15 (1965), 61-83.

10. J. R. Schue, The structure of hyperreducible triangular algebras, Proc. Amer. Math. Soc. 15 (1964), 766-772.

11. J. L. Orr, Diagonal disjoint ideals of nest algebras, Ph. D. thesis, King's College, University of London, 1989.

Department of Mathematics, Aegean University, Karlovasi 83200, Greece

Section of Mathematical analysis and its Applications, Department of Mathematics, University of Athens, Panepistimioupolis, 15784 Athens, Greece 